\title{
Spin dynamics and Griffiths singularity in the random quantum Ising magnet $\mathrm{PrTiNbO}_{6}$
}

\author{
Yuesheng Li $\mathbb{D}^{1,2 凶}$, Qiao-Yi Li ${ }^{3}$, Wei Li $\mathbb{D}^{3,4 凶}$, Tao Liu ${ }^{5}$, David J. Voneshen $\mathbb{D}^{6,7}$, Pabitra K. Biswas (iD) and Devashibhai Adroja ${ }^{7,8}$
}

In crystalline magnets, interaction randomness is usually thought as a negative factor preventing interesting quantum phenomena to occur. However, intriguing interplay between randomness and quantumness can also leads to unique phenomena in the strongly correlated materials. Among others, the random transverse-field Ising spin chain (RTIC) hosts a renowned quantum Griffiths phase. Although the RTIC model has been regarded as a toy model for long, here we materialize this model with the compound $\mathrm{PrTiNbO}_{6}$, which has a disordered ground state with pronounced quantum fluctuations and continuous spin excitations. The observed anomalous spin dynamics of PrTiNbO$_{6}$ can be accounted by the RTIC model with a consistent set of parameters determined from fitting the thermodynamic data, and it is ascribed to the quantum Griffiths rare regions in the system. Our results provide a concrete example of quantum Griffiths magnet, and offer an ideal experimental platform for investigating the dynamical properties of random many-body system.

npj Quantum Materials (2021)6:34; https://doi.org/10.1038/s41535-021-00333-6

\section{INTRODUCTION}

Randomness in interactions caused by imperfection is inevitable in solid-state materials. The presence of randomness is commonly believed to be "harmful", i.e., it may weaken the symmetrybreaking order and/or destabilize the quantum critical point at low dimensions ${ }^{1,2}$. Nevertheless, it also gives rise to exotic quantum phases and phenomena that are unique in quenched disorder systems ${ }^{3-9}$. For example, the exotic Griffiths phase can emerge in the random systems, which is characterized by strong power-law singularity in statical and dynamical properties ${ }^{8,10,11}$.

The prototypical and minimal model for understanding the quantum Griffiths phase (QGP) is the random transverse-field Ising chain (RTIC), where spin couplings and transverse fields are not uniform but follow a distribution amongst different sites/bonds ${ }^{8,9}$. The quantum critical point in uniform quantum Ising chain is strongly modified by the random couplings and fields, into to a quantum Griffiths criticality with divergent dynamical exponent, and the conventional paramagnetic phase turned into a peculiar disordered one with off-critical singularity ${ }^{12-15}$. The exotic quantum Griffiths singularity, with no counterpart in clean systems, has been reported in experiments, including the ferromagnetic alloy $\mathrm{Ni}_{1-x} \mathrm{~V}_{\mathrm{x}}{ }^{16,17}$, thin gallium superconducting films ${ }^{18}$, and has been predicted in the randomly layered superfluids $^{19,20}$, etc. In these examples, the QGP and related singular behaviors were analyzed phenomenologically, yet the complication of these systems hinders a concise microscopic theory for the presence of QGP. Therefore, finding a RTIC model material would then be of great significance and thus provide an unambiguous example and versatile platform studying the quantum Griffiths physics.

However, unlike the uniform Ising chain that has been materialized in crystalline compounds, including, e.g., $\mathrm{CoNb}_{2} \mathrm{O}_{6}{ }^{21}$ and $\mathrm{Sr}(\mathrm{Ba}) \mathrm{Co}_{2} \mathrm{~V}_{2} \mathrm{O}_{8}{ }^{22}$, the RTIC model remains a toy model and its experimental realization has been absent for decades. A key challenge for the realization of the RTIC model is to introduce randomness in both transverse fields and couplings. It makes the RTIC model, albeit simple, barely realized in real materials. For example, in the diluted rare-earth Ising magnet, $\mathrm{LiHo}_{x} \mathrm{Y}_{1-x} \mathrm{~F}_{4}$, there are random couplings present ${ }^{23-26}$, while the transverse magnetic field is always external and virtually uniform. Similarly, there is also no quantum Griffiths physics reported in the Ising-like quasi-spinchain antiferromagnet $\mathrm{Sr}(\mathrm{Ba}) \mathrm{CO}_{2} \mathrm{~V}_{2} \mathrm{O}_{8}$ under an applied transverse field ${ }^{27-30}$. On the other hand, although the ground state, as well as thermodynamics of the RTIC model, are relatively well understood theoretically $8,9,12-15$, the dynamical properties of the quantum Griffiths phase remains largely unexplored ${ }^{7}$.

In this article, we remove the obstacle by uncovering that the rare-earth compound $\mathrm{PrTiNbO}_{6}$ experimentally realizes the RTIC model, through thermodynamic and dynamical measurements on the single-crystal samples. The intrinsic transverse fields in $\mathrm{PrTiNbO}_{6}$ stem from the energy splittings between the two lowest-lying crystal-electric-field (CEF) levels of $\mathrm{Pr}^{3+}$ ions. As the nonmagnetic $\mathrm{Ti}^{4+}$ and $\mathrm{Nb}^{5+}$ ions randomly take the same $8 d$ Wyckoff position ${ }^{31}$, the CEF splittings follow a distribution and give rise to both random transverse fields and Ising couplings. We perform comprehensive dynamical measurements, including muon spin relaxation ( $\mu \mathrm{SR})$ and inelastic neutron scattering (INS). We find, besides the power-law thermodynamics, that the quantum Griffiths singularity is also evidenced by the pronounced spin fluctuations and continuous spin excitations at low temperatures. It turns out the RTIC model, with the parameters determined from fitting the thermodynamic data, can naturally explain the dynamical properties of $\mathrm{PrTiNbO}_{6}$. The many-body modeling also enables us to nail down the origin of the continuous spin

\footnotetext{
${ }^{1}$ Wuhan National High Magnetic Field Center \& School of Physics, Huazhong University of Science and Technology, Wuhan, China. ${ }^{2}$ Experimental Physics VI, Center for Electronic Correlations and Magnetism, University of Augsburg, Augsburg, Germany. ${ }^{3}$ Key Laboratory of Micro-Nano Measurement-Manipulation and Physics (Ministry of Education), School of Physics, Beihang University, Beijing, China. ${ }^{4}$ International Research Institute of Multidisciplinary Science, Beihang University, Beijing, China. ${ }^{5}$ School of Science, Hunan University of Technology, Zhuzhou, China. ${ }^{6}$ Department of Physics, Royal Holloway University of London, Egham, UK. ${ }^{7}$ ISIS Pulsed Neutron and Muon Source, STFC Rutherford Appleton Laboratory, Harwell Campus, Didcot, Oxfordshire, UK. ${ }^{8}$ Highly Correlated Matter Research Group, Physics Department, University of Johannesburg, Auckland Park,

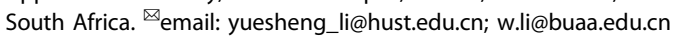



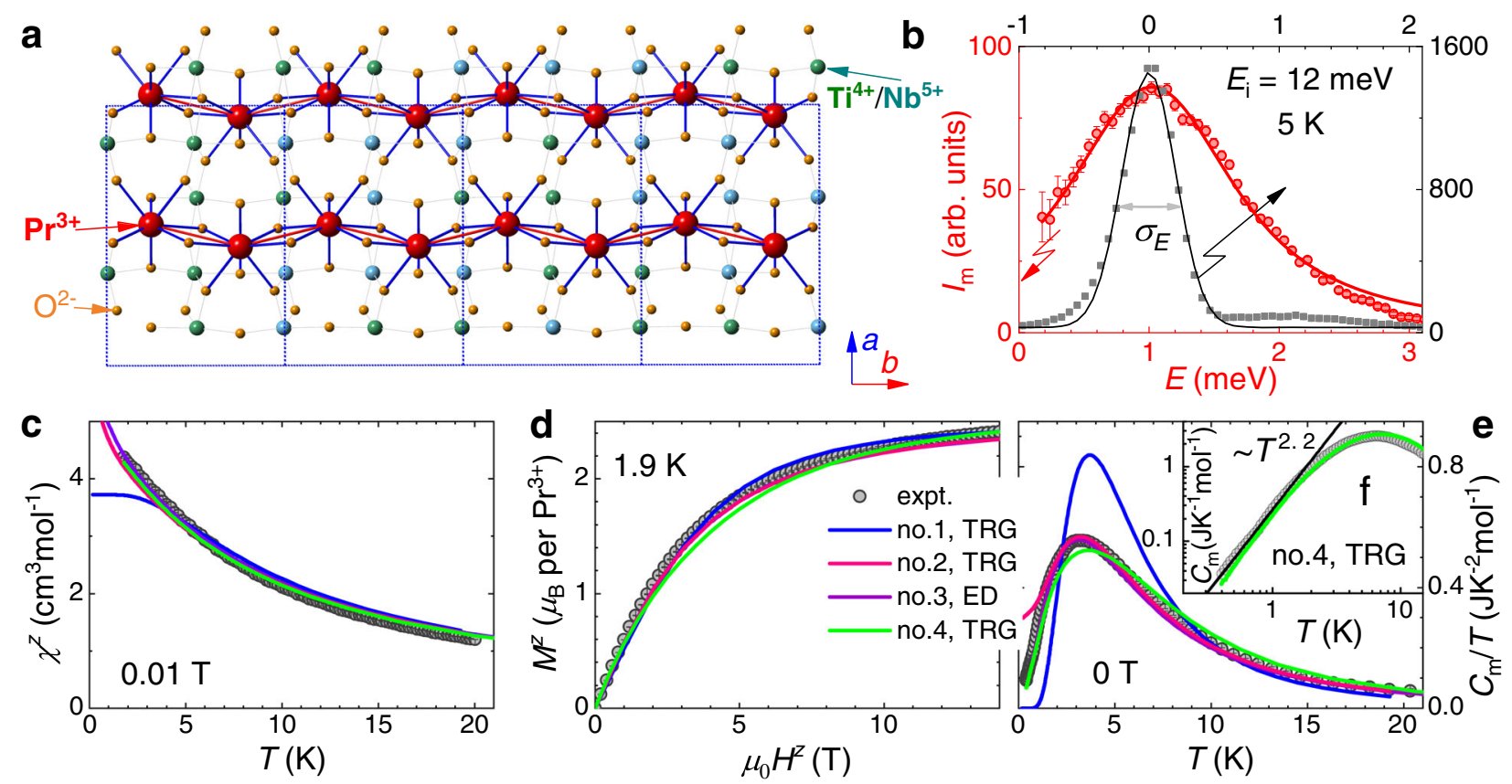

Fig. 1 Crystal structure, CEF splitting, and thermodynamic fittings of PrTiNbO $_{6}$. a Crystal structure of $\mathrm{PrTiNbO}_{6}$ viewed in the $a b$ plane, where the dashed blue lines indicate the unit cells. b Magnetic INS data (red), where the elastic and lattice INS contribution measured on the nonmagnetic reference compound, $\mathrm{LaTiNbO}_{6}$, has been subtracted. The gray squares present the total intensity of PrTiNbO 6 . The gray line shows the Gaussian fit to the elastic signal with the fitted resolution of $\sigma_{E}=0.48(1)$ meV, whereas the red line presents the fit to the magnetic data with the convolution function (see main text). c-e Combined fits to measured thermodynamic data ${ }^{31}$ using models no. 1 (without randomness) and 2-4 (random Ising chain with similar parameters, see main text). $f$ Power-law temperature dependence of the magnetic heat capacity below $\sim 2 \mathrm{~K}$ measured at zero field, as compared to the green line calculated by model no. 4 . The lattice heat capacity measured on $\mathrm{LaTiNbO}_{6}$ has been subtracted in $(\mathbf{e}, \mathbf{f})^{31}$. Error bars on the experimental data points show a standard error.

spectra in the compound as the locally ordered quantum Griffiths rare region.

\section{RESULTS}

\section{Rare-earth random quantum Ising magnet}

The structure of spin-chain material $\mathrm{PrTiNbO}_{6}$ is shown in Fig. $1 \mathrm{a}$, where the $\mathrm{Pr}^{3+}$ ions contribute effective Ising magnetic moments owing to the two lowest-lying CEF singlets, $\left|E_{1}\right\rangle$ and $\left|E_{2}\right\rangle^{31}$. In the subspace of $\left|E_{1}\right\rangle$ and $\left|E_{2}\right\rangle$, the matrix element of the magnetic moment is given by $m_{j, j^{\prime}}{ }^{a}=g_{J} \mu_{\mathrm{B}}\left\langle E_{j}\left|J^{a}\right| E_{j^{\prime}}\right\rangle\left(a=x, y, z\right.$, and $\left.j, j^{\prime}=1,2\right)$, where $g_{J}=4 / 5$ is the Landé $g$ factor. At low temperatures $\left(k_{\mathrm{B}} T<<\left\langle E_{3}-E_{1}\right\rangle\right.$, see below), the components of the effective Ising magnetic moments of $\mathrm{Pr}^{3+}$ are naturally obtained,

$\mathbf{m}^{\mathbf{x}}=\mathbf{m}^{\mathbf{y}}=\left(\begin{array}{ll}0 & 0 \\ 0 & 0\end{array}\right), \mathbf{m}^{\mathbf{z}}=\frac{\mu_{\mathrm{B}}}{2}\left(\begin{array}{cc}0 & G \\ G^{*} & 0\end{array}\right)$

where $|G|=g \sim 5$ is the effective pseudospin-1/2 $g$ factor along the $c(z)$ axis in $\mathrm{PrTiNbO}_{6}$. The eigenstates of Eq. (1) are the effective pseudospin-1/2 states,

$$
\left|S^{z}= \pm 1 / 2\right\rangle=\frac{1}{\sqrt{2}}\left(\frac{G}{|G|}\left|E_{1}\right\rangle \pm\left|E_{2}\right\rangle\right)
$$

with the Ising eigen-moments, $m^{x}=m^{y}=0$ and $m^{z}= \pm \mu_{\mathrm{B}} g / 2$. The dipolar magnetic moments of $\mathrm{Pr}^{3+}$ is restricted along the $c$ axis (Ising anisotropy), while the $x y$-plane moments are mostly multipolar. Therefore, the external magnetic field only couples to the $z$ component of the pseudospin-1/2 moments, as seen in the magnetization measurements ${ }^{31}$. Furthermore, since the two lowest-lying CEF singlets of $\left|E_{1}\right\rangle$ and $\left|E_{2}\right\rangle$ form a non-Kramers quasidoublet, the level splitting $h^{x} \equiv E_{2}-E_{1}$ constitutes an effective transverse-field term in the pseudospin-1/2 Hamiltonian ${ }^{32-34}$, i.e.,

$$
\mathcal{H}_{S I}=\frac{E_{2}-E_{1}}{2}\left(\left|E_{2}\right\rangle\left\langle E_{2}|-| E_{1}\right\rangle\left\langle E_{1}\right|\right)=-h_{j}^{x} S_{j}^{X},
$$

where $h_{j}^{x}$ for site $j$ follows a distribution as evidenced by the INS measurements (see below).

In Fig. $1 \mathrm{~b}$, the first CEF excitation is probed by INS at $5 \mathrm{~K}$ and is found much broader than the instrumental resolution of $\sigma_{E}=0.48(1)$ $\mathrm{meV}$. Fitting the broad peak with the convolution function ${ }^{32,35}$, $I_{\mathrm{m}}(E)=\int \frac{2 l_{0}}{\pi \sigma_{E}} \frac{\omega_{\mathrm{L}}}{4\left(E /-\left\langle E_{2}-E_{1}\right\rangle\right)^{2}+\omega_{L}^{2}} \sqrt{\frac{4 \ln 2}{\pi}} \exp \left(-\frac{4 \ln 2(E-E)^{2}}{\sigma_{E}^{2}}\right) d E \prime$, we find a Lorentzian distribution of $h_{j}^{x}$ which has a mean value $\left\langle E_{2}-E_{1}\right\rangle \sim 1.0 \mathrm{meV}$ and a full width at half maxima (FWHM) $\omega_{\mathrm{L}} \sim 1.4 \mathrm{meV}$, where $I_{0}$ is the integral intensity. This is in good consistency with our previous estimate based on the analysis of the heat capacity of $\operatorname{Pr}_{0.08} \mathrm{La}_{0.92} \mathrm{TiNbO}_{6}{ }^{31}$. The next CEF excited energy is much larger, i.e., $\left\langle E_{3}-E_{1}\right\rangle \sim 11.9 \mathrm{meV} \sim 140 \mathrm{~K}^{31}$. Henceforth, we focus on the lowest quasi-doublet below $\sim 20 \mathrm{~K}$, and safely ignore higher CEF levels. Besides, there exists antiferromagnetic (AF) Ising coupling d) $\sim 4 \mathrm{~K}$ between the pseudospin-1/2 moments via the " $\operatorname{Pr}(f)-\mathrm{O}(p)-\operatorname{Pr}$ $(f)^{\prime \prime}$ superexchange path ${ }^{31,36,37}$. As the bond coupling $J_{j}$ depends on the wavefunctions of the lowest-lying quasi-doublets at adjacent sites ${ }^{36,37}$, it is also randomly distributed in $\operatorname{PrTiNbO}_{6}$.

\section{Determination of the RTIC model parameters}

As the Ising moments in $\mathrm{PrTiNbO}_{6}$ are coupled through random couplings and under intrinsic random transverse fields, the microscopic RTIC Hamiltonian for the compound reads,

$\mathcal{H}=\sum_{j=1}^{I} J_{j} S_{j}^{z} S_{j+1}^{z}-\sum_{j=1}^{I} h_{j}^{x} S_{j}^{x}-\mu_{0} H^{z} \mu_{B} \sum_{j=1}^{I} g_{j} S_{j}^{z}$,

where $J_{j}$ represents Ising coupling strength between $\mathrm{Pr}^{3+}$ ions $\left(S_{j}^{z}\right.$ moments), and $H^{z}$ is the external longitudinal magnetic field. 

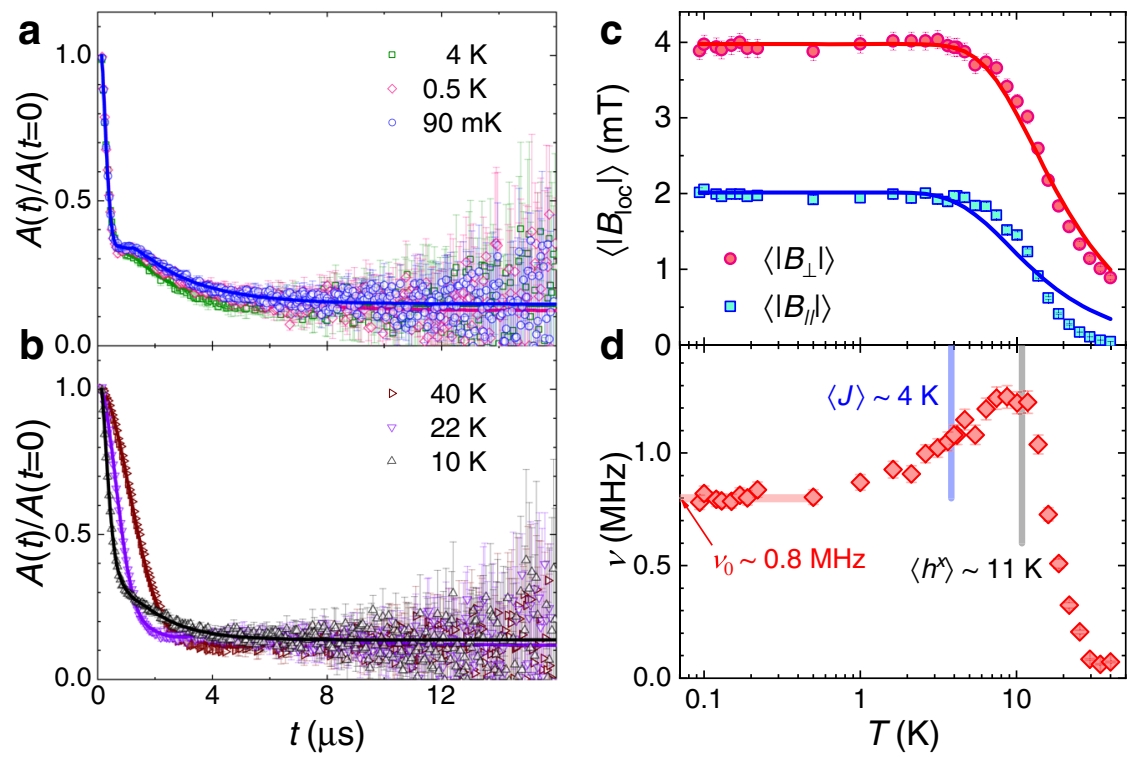

Fig. $2 \mu$ SR measured on the single crystal of PrTiNbO $_{6}$. $\mathbf{a}$, b Selected ZF- $\mu$ SR spectra with the incident beam perpendicular to the $c$ axis in the dilution refrigerator and ${ }^{4} \mathrm{He}$ cryostat, respectively. The colored lines represent the Gaussian DKT fits. c Temperature dependence of the average absolute value of the local-field components perpendicular $\left(\left\langle\left|B_{\perp}\right|\right\rangle\right)$ and parallel $\left(\left\langle\left|B_{||}\right|\right\rangle\right)$to the incident beam, with the colored lines showing the fits (see main text). d Temperature dependence of the fluctuation rate of the local field around the implanted $\mu^{+}$. Error bars on the data points show a standard error.

Other symmetry-allowed terms like $\left(S_{j}^{+} S_{j+1}{ }^{+}+\right.$h.c.) are expected to be small $(<1 \mathrm{~K})^{31}$ and not included in constructing our minimal RTIC model for PrTiNbO

To accurately determine the parameters in pseudospin-1/2 Hamiltonian (Eq. (4)) of $\mathrm{PrTiNbO}_{6}$, we fit the thermodynamic data using four different models (Fig. 1c-e). In model no. 1, we neglect the randomness, and find an optimal parameter set $J=3.5 \mathrm{~K}, h^{x}=$ $12 \mathrm{~K}$, and $g=5.0$ within the uniform spin chain model, which, however, fails to fit the thermodynamic data well (see Fig. 1), quantified by a rather large residual $R_{p}=35$ (see Eq. (8) for the definition of $R_{p}$ ). It thus confirms the indispensable ingredient of randomness in $\mathrm{PrTiNbO}_{6}$.

Therefore, we introduce independent Lorentzian distribution of $J, h^{x}$, and $g$ (as the lineshape of the first CEF excitation indicates in Fig. $1 \mathrm{~b}$ ) in the models no. $2-3$, and find their optimal values by fitting the experimental thermodynamic data. In model no. 2, tensor renormalization group (TRG) calculations are performed on rather long spin chains with length $I=1000$, averaged over 20 sample chains, which leads to a very accurate estimate of parameters as $J=3.5 \mathrm{~K}, \Delta=3.1 \mathrm{~K}$ ( $\Delta$ represents the FWHM of distribution, same below), $\left\langle h^{x}\right\rangle=12 \mathrm{~K}, \Delta h^{x}=11 \mathrm{~K},\langle g\rangle=5.0$, and $\Delta g=0.05$. The fitting with model no. 2 is excellent above $1.5 \mathrm{~K}$, with significantly reduced least $-R_{p} \sim 10$. In no. 3 model, we perform exact diagonalization (ED) calculations of $I=12$ chains averaged over 48 samples, and get $\left\langle J=3.8 \mathrm{~K}, \Delta J=2.8 \mathrm{~K},\left\langle h^{x}\right\rangle=\right.$ $11 \mathrm{~K}, \Delta h^{x}=10 \mathrm{~K},\langle g\rangle=5.0$, and $\Delta g=0.06$ with the least $R_{p}=8.6$. Moreover, to further confirm the microscopic RTIC model for the compound, in model no. 4 we take a fixed distribution of $\left\{h_{j}^{x}\right\}$ measured by LET at $9 \mathrm{~K}$ with a high resolution (Supplementary Note 2) that is slightly asymmetric. We then can fit the thermodynamic data down to $0.4 \mathrm{~K}$, by fine tuning other parameters, and obtain \langle\rangle$=3.5 \mathrm{~K}, \Delta J=11 \mathrm{~K},\langle g\rangle=4.9$, and $\Delta g=$ 0.05 , with $R_{p}=14$.

Overall, from the fittings with random Ising models no. 2-4, we obtain a reliable and consistent estimate of random couplings and fields in $\mathrm{PrTiNbO}_{6}$. In particular, we find $\left.\left\langle h^{x}\right\rangle / J\right\rangle\left(\right.$ or $\left.\Delta h^{x} / \Delta\right) \sim 3$ is much larger than the critical point of $1 / 2$ (We use the spin operators, $S=\sigma / 2$, in the RTIC Hamiltonian (Eq. (4)), and thus there exists a pre-factor of $1 / 2$ in front of the critical value reported in, e.g., ref. ${ }^{12}$, where Pauli matrices $\sigma$ were taken.), located deeply in the disordered QGP regime. Nevertheless, due to interaction randomness, there exists quantum Griffiths singularity in the lowtemperature scalings of thermodynamics. The susceptibility rapidly increases as $T$ lowers, and the magnetic specific heat exhibits an algebraic behavior below $2 \mathrm{~K}$. The latter indicates a nonzero dynamical exponent $z \sim 0.45$ characterizing the quantum Griffiths singularity, which otherwise should be zero if the transverse fields were uniform.

\section{Quantum spin fluctuations}

Zero-field (ZF) $\mu \mathrm{SR}$ is an extremely sensitive probe detecting the fluctuations of internal local fields in magnetic materials. Figure $2 a, b$ shows general Gaussian dynamic Kubo-Toyabe (DKT) relaxations ${ }^{38}$ (see the Supplementary Note 3), due to the relatively large distribution of the local magnetic fields induced by the strong hyperfine couplings of ${ }^{141} \mathrm{Pr}^{3+}{ }^{39,40}, \gamma_{\mu}\left|B_{\text {loc }}\right|>\sim V_{1}$, where $\gamma_{\mu}=$ $135.5 \mathrm{MHz} / \mathrm{T}$ is the $\mu^{+}$gyromagnetic ratio, $B_{\text {loc }}$ is the local magnetic field around the implanted $\mu^{+}$, and $v$ is the fluctuation rate. As shown in Fig. $2 c$, the local fields, perpendicular $\left(\left\langle B_{\perp} \mid\right\rangle\right)$ and parallel $\left(\left\langle B_{\|} \mid\right\rangle\right)$to the incident $\mu^{+}$beam, are found to have distribution widths in the order of $\sim 1 \mathrm{mT}$, similar to other quantum disordered Pr-based magnets ${ }^{39,40}$. We further fit to the temperature dependence of the average absolute value of the local field using $\left\langle\mid B_{\mathrm{loc}}\right\rangle \propto \tanh \left[\left\langle h^{x}\right\rangle \mid\right.$ $\left.\left(2 k_{\mathrm{B}} T\right)\right]$ by ignoring the distribution of $h^{x}$ and spin-spin interactions ${ }^{39}$, and find $\left\langle h^{x}\right\rangle \equiv\left\langle E_{2}-E_{1}\right\rangle \sim 1.2-1.8 \mathrm{meV}$ (see Fig. $2 \mathrm{c}$ ). The average value of the intrinsic random transverse fields obtained from $\mu \mathrm{SR}$ measurements is consistent with the above INS results (Fig. 1b).

The zero-temperature limit of the fluctuation rate of the local field reflects the quantum fluctuations of spins, which are pronounced in quantum spin liquid candidates. For instance, a very strong quantum fluctuation rate of $\sim 9 \mathrm{MHz}$ has been reported in $\mathrm{YbMgGaO}_{4}$ at $\sim 0.07 \mathrm{~K}^{41,42}$. As shown in Fig. $2 \mathrm{~d}$, the zero-temperature fluctuation rate in $\mathrm{PrTiNbO}_{6}$ is $v_{0} \sim 0.8 \mathrm{MHz}$, indicating the existence of significant quantum fluctuations in the compound. Both the magnetically disordered bulk (medium) and the putative fluctuating rare regions under the tunneling fields (see below) can contribute to the dynamic $\mu$ SR signal. Notably, broad spin fluctuations have also been reported in the random system $\mathrm{Ni}_{1-x} \mathrm{~V}_{x}$ with quantum Griffiths singularity observed ${ }^{17}$. 

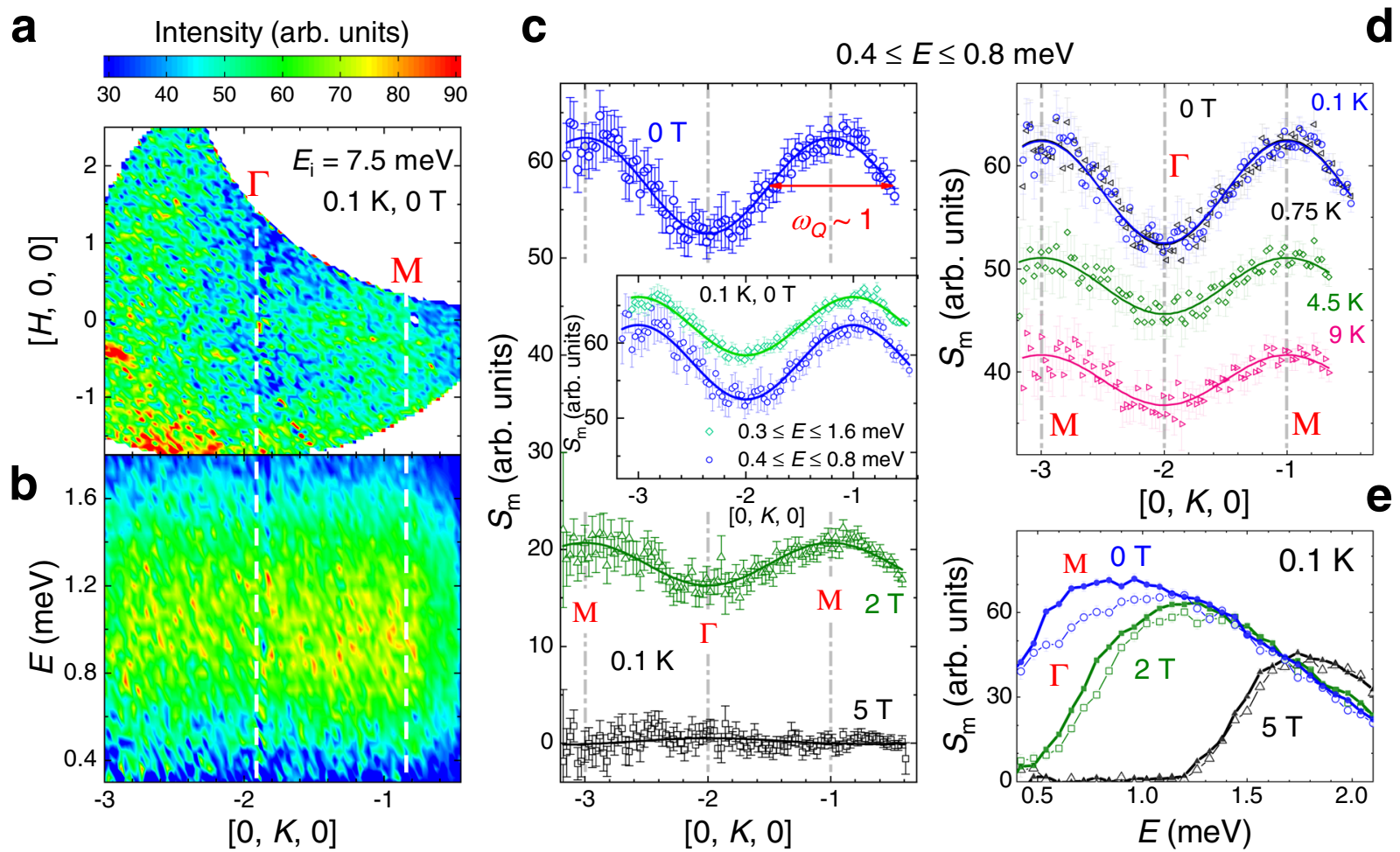

Fig. 3 INS spectra of the PrTiNbO ${ }_{6}$ single crystal measured with $E_{\mathrm{i}}=\mathbf{7 . 5} \mathbf{~ m e V}$. a Wave-vector dependence of the spin excitation continuum measured at $0.4 \leq E \leq 0.8 \mathrm{meV}$ at $0.1 \mathrm{~K}$. b Energy dependence of the continuum along the $b$ axis. $\mathbf{c}$, $\mathbf{d}$ Wave-vector dependence of the dynamic spin-correlation function measured at selected longitudinal fields at $0.1 \mathrm{~K}$ and measured at various selected temperatures under zero field, respectively. The colored lines are guide for the eye and the dashed gray lines mark the high-symmetry reciprocal-space points ( $\Gamma$ and $M$ points). In $\mathbf{c}$ the FWHM of the hump $\left(\omega_{Q}\right)$ is marked, and the inset shows the data integrated over different energy ranges. e Energy dependence of the measured intensities at the $M$ (solid scatters) and $\Gamma$ (open scatters) points, respectively. The $8.8 \mathrm{~T}$ data provides the elastic background below $2 \mathrm{meV}$, which has been subtracted in the INS data shown in $(\mathbf{a}-\mathbf{e})$. Error bars on INS data indicate one standard error propagated from neutron counts.

\section{Spin dynamics and excitation continuum}

Neutron scattering measurements provide valuable information on the dynamical spin correlations and excitations in quantum magnets ${ }^{43}$. For PrTiNbO $_{6}$, despite the AF Ising coupling of \langle\rangle$\sim 4 \mathrm{~K}$, no magnetic freezing is evidenced, as there is neither splitting of zero-field-cooling and field-cooling susceptibilities down to $1.8 \mathrm{~K}$, nor sharp " $\lambda$ " heat capacity peak down to $\sim 0.1 \mathrm{~K}$. Moreover, no frequency dependence of the ac susceptibilities was observed ${ }^{31}$. This is confirmed by the $\mu \mathrm{SR}$ and neutron scattering measurements in this work (see Figs. 2 and 3). As indicated by the columnar-like shape in Fig. 3a, we find the correlations are mainly along the $b$ axis (i.e., spin-chain direction) but not along the orthogonal $a$ axis, suggesting a negligible interchain correla$\operatorname{tion}^{32,33}$, due to relatively large interchain distance $(\geq 5.4 \AA)^{31}$.

In Fig. 3, we can observe a continuum of excitations broadly distributed in both momentum and energy space even at the lowest temperature of $0.1 \mathrm{~K}$, where the magnetic entropy is estimated to be almost zero ${ }^{31}$. As shown in Fig. 3e, the applied longitudinal magnetic field gradually shifts the spectral weight toward higher energies, thus indicating the magnetic origin of these excitations. Owing to the dominant single-ion physics, $\left.\left.\left\langle E_{2}-E_{1}\right\rangle \equiv\left\langle h^{x}\right\rangle\right\rangle\right\rangle\langle\rangle$, the wave-vector dependence of the spectra is negligible at $E>>\backslash$, which can be evidenced in Fig. $3 e$, while below $\sim 1 \mathrm{meV} \sim 3$ J) the wave-vector dependence of the dynamic spin correlation $S_{m}$ is significant. Therefore, in the analysis below, we focus on the energy range between 0.4 and $0.8 \mathrm{meV}$, and the energy integration of the entire continuum slightly weakens the wave-vector dependence in the integrated spin structure $S_{m}$ (see the inset of Fig. 3c).
There exist two magnetic $\mathrm{Pr}^{3+}$ ions per unit cell along the chain (see Fig. 1a), and the broad humps appear around the $M$ points, i.e., $K=-3,-1$, etc. (see Fig. $3 c$, d), suggesting the formation of $A F$ correlations in the system. By applying longitudinal field (e.g., $\sim 2 \mathrm{~T}$ ) or increasing temperature (e.g., $\sim 4.5 \mathrm{~K}$ ), the wave-vector dependence of $S_{m}$ as well as the overall intensity are clearly suppressed in $\mathrm{PrTiNbO}_{6}$ (see Fig. 3c, d). These observations indicate that the AF correlations, driven by the coupling $J$ of $\sim 4 \mathrm{~K}$, are mostly restricted within the nearest neighbors, i.e., with a short correlation length $\xi_{b} \sim b /\left(\pi \omega_{Q}\right) \sim 2.4 \AA$ (see Fig. $3 c$ for $\left.\omega_{Q}\right)^{44-46}$. Even for a low-energy transfer down to $\sim 0.05 \mathrm{meV}$, the peak of the INS spectrum (around the $M$ point) remains broad in $\mathrm{PrTiNbO}_{6}$ (Supplementary Note 1). This is even quite different from other disordered magnets, e.g., $\mathrm{YbMgGaO}_{4}{ }^{42,46}$, where the correlation length increases at lower transfer energies. We ascribe the difference to the intrinsic random effects of the non-Kramers magnet $\mathrm{PrTiNbO}_{6}$ (see below).

\section{RTIC dynamics and quantum Griffiths rare region}

With the RTIC models (no. 1-4) determined from fitting thermodynamic properties, in Fig. 4 we compute the dynamical spin structure factor and spectral weight of the RTIC model, and compare directly to experimental measurements. Firstly, model no. 1 without randomness again fails to capture the $E$ and $K$ dependence of dynamical spectra. The uniform chain exhibits a narrow magnon "band" in the $E$ dependence (see Fig. 4i), and sharp peaks in spin structure factor at $K=-3,-1$ in Fig. 4g, clearly different from the experimental measurement at low temperatures. On the other hand, models no. 2-4, with similar random parameters (see above), which can fit the thermodynamics very 
a
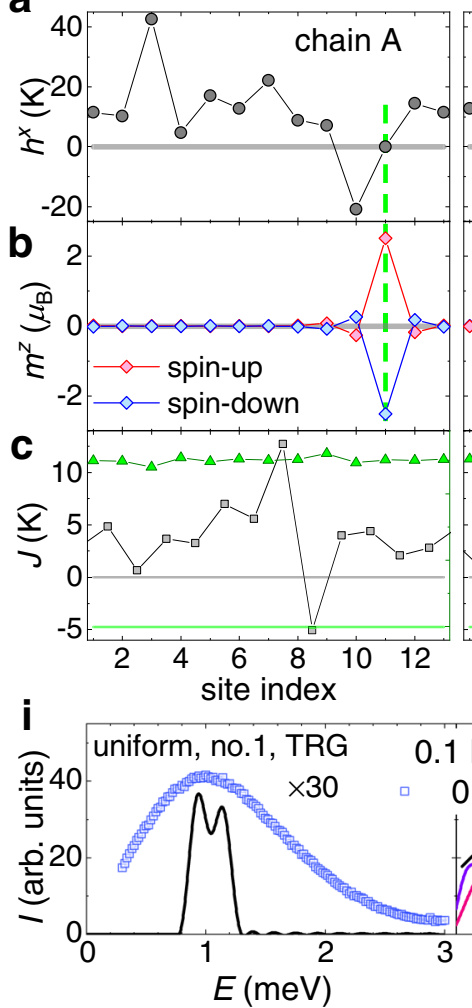
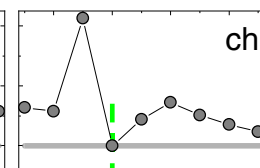

chain B
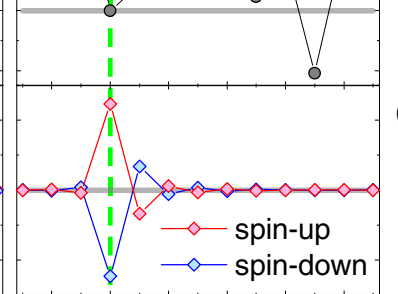

e

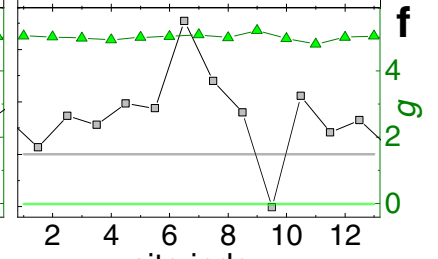

$0.1 \mathrm{~K}$
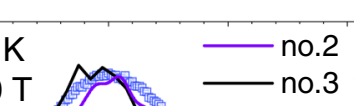

- no. 2

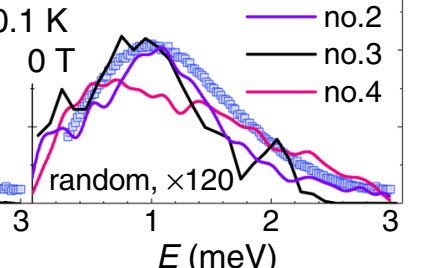

d $\mathrm{g}$

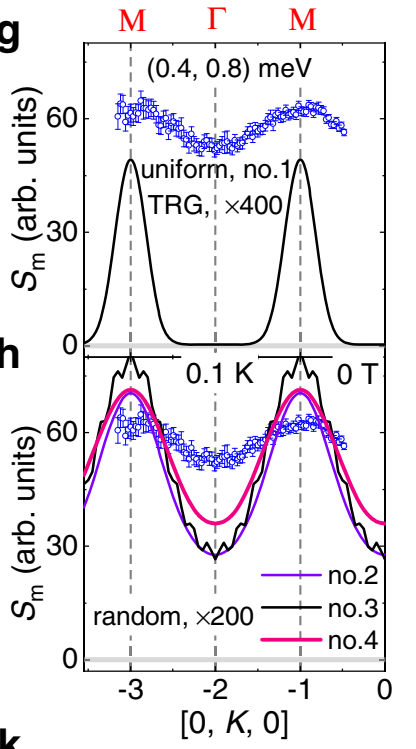

$\mathbf{k}$

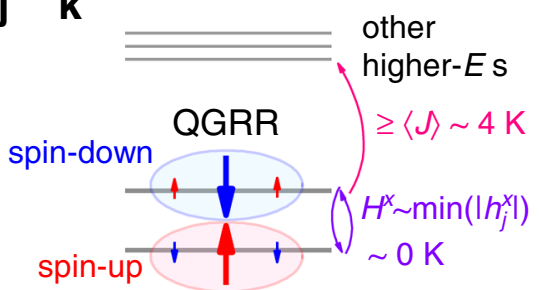

Fig. 4 Calculated ground state and dynamical properties of PrTiNbO $_{6}$. a, c Transverse field distribution, and effective $g$ factors of each $\mathrm{Pr}^{3+}$ ion, together with the distribution of the Ising couplings, for the sample chain A. b Calculated local magnetic moments and related spinup and -down states of QGRR in the chain A. $\mathbf{d}-\mathbf{f}$ Corresponding plots of the sample chain B. $\mathbf{g}, \mathbf{h}$ Dynamical structure factors are calculated using the uniform chain model no. 1 and the random models no. $2-4$, respectively. $\mathbf{i}$, $\mathbf{j}$ The measured energy dependence of the INS intensities $\left(E_{\mathrm{i}}=3.7 \mathrm{meV}\right)$, with the colored lines showing the calculated data of models no. 1-4. Error bars on INS data indicate one standard error propagated from neutron counts. A more detailed comparison between experimental and numerical data under applied fields can be found in Supplementary Note 2. k Illustration of the energy spectrum where the two-lowest levels represent a QGRR.

well (see Fig. 1) can also semi-quantitatively produce the spin spectra in Fig. 4h, j. The anomalous excitation continuum observed in experiments, including the large density of states at small energies and broad peaks in $S_{\mathrm{m}}$ vs. $K$, are well produced by our RTIC models no.2-4, without any further adjustment of the interaction parameters (see Fig. $4 \mathrm{~h}, \mathrm{j}$ ).

Given the accurate RTIC modeling of PrTiNbO $_{6}$, we can now analyze the origin of quantum Griffiths singularity in the compound. In Fig. 4, we show the ED calculations of magnetic moments along the spin chain in two typical samples, with the site-dependent parameters $J_{j}, h_{j}^{X}, g_{j}$ generated from the Lorentzian distribution of model no. 3 . In each sample chain, we find two lowest-lying states, $\mid E_{1}^{\text {tot }}$, and $\mid E_{2}^{\text {tot }}>$ that are nearly degenerate and with a small energy splitting (inner gap) proportional to the minimal absolute value amongst all random fields, $\min \left(\left|h_{j}^{x}\right|\right)$. This collective quasi-doublet is well separated from higher-energy states by a relatively large gap of $\geq \downarrow\rangle \sim 4 \mathrm{~K}$ (Supplementary Note 2). Similar to Eq. (2), at low temperatures $(T<\langle J\rangle)$ we can reconstruct the spin-up and spin-down states out of $\left|E_{1}{ }^{\text {tot }}\right\rangle$ and $\mid E_{2}{ }^{\text {tot }}$, through a coherent superposition,

$\left|S_{\mathrm{tot}}^{z}= \pm 1 / 2\right\rangle=\frac{1}{\sqrt{2}}\left(\left|E_{1}^{\mathrm{tot}}\right\rangle \pm\left|E_{2}^{\mathrm{tot}}\right\rangle\right)$.

The two orthonormal states, $\left|S_{\text {tot }}{ }^{z}= \pm 1 / 2\right\rangle$, as illustrated in Fig. 4k, have a nonzero net moment restricted within the quantum Griffiths rare region (QGRR) ${ }^{5-7}$ well localized in a RTIC and thus can be regarded as a collective "qubit".
By resetting $\left(E_{1}{ }^{\text {tot }}+E_{2}{ }^{\text {tot }}\right) / 2=0$, we write down an effective Hamiltonian for such a qubit under the external field $H^{z}$,

$\mathcal{H}_{\mathrm{QGRR}}=-H^{\mathrm{x}} S_{\text {tot }}^{\mathrm{x}}-\mu_{0} H^{z} \mu_{B} g_{\text {tot }} S_{\text {tot }}^{z}$,

where $g_{\text {tot }}=2\left|\sum g_{j}\left\langle S_{\text {tot }}^{z}= \pm 1 / 2\left|S_{j}^{z}\right| S_{\text {tot }}^{z}= \pm 1 / 2\right\rangle\right|$ is the overall effective $g$ factor, and $H^{x} \equiv E_{2}{ }^{\text {tot }}-E_{1}{ }^{\text {tot }}$ enables quantum tunneling between spin up and down, and $S_{\text {tot }}{ }^{x} \equiv\left(\mid S_{\text {tot }}{ }^{z}=1 / 2 \times S_{\text {tot }}{ }^{z}=\right.$ $\left.-1 / 2|+| S_{\text {tot }}^{z}=-1 / 2 \times S_{\text {tot }}^{z}=1 / 2 \mid\right) / 2$ is highly similar to $S_{j}^{x}$ in Eq. (3). The resulting distribution of local magnetic moments, $\left\langle m_{j}^{z}\right\rangle_{ \pm}=$ $g_{j}\left\langle S_{\text {tot }}{ }^{z}= \pm 1 / 2\left|S_{j}^{z}\right| S_{\text {tot }}{ }^{z}= \pm 1 / 2\right.$, are shown in Fig. $4 \mathrm{~b}$ for the sample chain $A$ and in Fig. 4 e for the sample chain $B$, from which we find that each QGRR has nonzero moments centered at $h_{j}^{x} \sim 0 \mathrm{~K}$ (indicated by the green dashed lines). The AF correlation extends to only few neighbors, while rest spins of the chain remain disordered, i.e., $\left\langle m_{j}^{z}\right\rangle_{ \pm} \sim 0$, due to the relatively large transverse field $\left\langle h^{x}\right\rangle$.

The QGRR, a correlated local cluster, contributes a nonzero net magnetic moment, i.e., $g_{\text {tot }}>0$, to the random quantum Ising chain. The QGRR is responsible for the observed Griffiths singularity ${ }^{7}$ evidenced by the low- $T$ diverging susceptibility (Fig. 1c), as well as reflected in the broad continuous spin excitations (Fig. 4) in PrTiNbO $_{6}$. The latter contributes a virtually uniform background beneath the cosine-like dispersion due to short-range AF correlations. In contrast, the model no. 1 without randomness, and thus without QGRR, cannot reproduce the singular thermodynamic behaviors (Fig. 1c, e) and the spin excitation continuum (Fig. $4 \mathrm{~g}$, i). 


\section{DISCUSSION}

Overall, it is very remarkable that all the measured equilibrium thermodynamic properties of $\mathrm{PrTiNbO}_{6}$ can be captured accurately by this simple, yet profound, quantum RTIC model, with a proper set of random parameters. Moreover, the model also captures the main features of the spin excitation continuum. Given the accurate microscopic spin model, we can well understand the anomalous spin excitations measured in the compound and ascribe them to the existence of QGRR.

Nevertheless, the real material is always more complicated than the effective model (see Eq. (4)), and there may exist other symmetry-allowed interaction terms and additional microscopic details in $\mathrm{PrTiNbO}_{6}$ that we did not fully take into account. For example, the asymmetric distribution of random transverse fields in model no. 4 can reproduce very well the low-temperature properties below $1 \mathrm{~K}$ (see Fig. 1f), suggesting some deviation from symmetric Lorenzian distribution of randomness in model no. 2 and 3 may further improve their fittings to $\mathrm{PrTiNbO}_{6}$ measurements. Besides, the inclusion of small non-RTIC interactions in $\mathrm{PrTiNbO}_{6}$ may also take effects below $1 \mathrm{~K}$. To understand their effects, we have computed the RTIC model under a small random XY perturbation $\left(S_{j}^{+} S_{j+1}{ }^{-}+\right.$h.c. $)$and observed that the quantum Griffiths singularity is intact (see Supplementary Note 4). Therefore, we believe that the RTIC model well captures the essential quantum Griffiths physics in the strongly correlated quantum material $\mathrm{PrTiNbO}_{6}$.

Lastly, as a unique phenomenon in the random spin systems that do not have clean counterparts, the quantum Griffiths singularity found in $\mathrm{PrTiNbO}_{6}$ can also exist in other non-Kramers rare-earth random compounds with correlated ground-state CEF quasi-doublets. One such example is the triangular-lattice Ising magnet, $\mathrm{TmMgGaO}_{4}{ }^{32,33,47}$, where similar site-mixing disorder of $\mathrm{Mg}^{2+} / \mathrm{Ga}^{3+}$ was reported and the randomness of the transverse field was also evidenced. Therefore, our present discovery in $\mathrm{PrTiNbO}_{6}$ sheds light on similar Pr- and Tm-based compounds with site mixing, which may constitute a family of non-Kramers quantum Griffiths magnets to be explored.

\section{METHODS}

\section{Crystal synthesization and experimental measurements}

Large single crystals $(\sim 1 \mathrm{~cm})$ of $\mathrm{PrTiNbO}_{6}$ were grown by the optical floating zone technique reported previously ${ }^{31}$. The $\mu$ SR data were collected on the MUSR spectrometer at the ISIS pulsed muon facility, Rutherford Appleton Laboratory, United Kingdom, with the incident muon beam along the $b$ axis (perpendicular to the Ising direction) between 0.09 and $4.2 \mathrm{~K}$ in a dilution refrigerator ${ }^{48}$. The additional data between 2 and $40 \mathrm{~K}$ were collected by transferring the sample to a ${ }^{4} \mathrm{He}$ cryostat. Moderatehigh-energy INS data were collected on the MERLIN spectrometer at the ISIS pulsed thermal neutron facility, Rutherford Appleton Laboratory, U.K., at $5 \mathrm{~K}$ using the powder samples of $\mathrm{PrTiNbO}_{6}(10.91 \mathrm{~g})$ and $\mathrm{LaTiNbO}_{6}$ $(10.85 \mathrm{~g})$. Two incident energies, $E_{\mathrm{i}}=12$ and $120 \mathrm{meV}$ were used with the instrumental resolutions of $\sim 0.5$ and $5 \mathrm{meV}$, respectively ${ }^{49}$.

Low-energy-transfer INS measurements were carried out on a cold neutron multi-chopper spectrometer LET at the ISIS pulsed neutron and muon source. Incident energies of 22.5, 7.5, 3.7, and $2.2 \mathrm{meV}$ were used with the energy resolutions of $1,0.22,0.11$, and $0.04 \mathrm{meV}$, respectively ${ }^{50}$. The sample temperature down to $0.1 \mathrm{~K}$ was achieved using a dilution refrigerator. Magnetic fields of $0,2,5$, and $8.8 \mathrm{~T}$ were applied along the $c$ axis. All datasets in Fig. 3 were integrated over the momentum space, $-0.3 \leq L \leq 0.3$ in $[0,0, L]$, the data in Fig. 3b-e were further integrated over the momentum space, $-0.3 \leq H \leq 0.3$ in $[H, 0,0]$. Moreover, the data at $\Gamma$ and $M$ points in Fig. 3e were further integrated over $-2.1 \leq K \leq-1.9$ and $-1.1 \leq K \leq-0.9$, respectively, in $[0, K, 0]$. All measured momenta were integrated over in Fig. 4i, j. The measured dynamic spin-correlation function is obtained from the raw intensity $\mathrm{as}^{43}$,

$S_{\mathrm{m}}(\mathbf{Q}, E) \sim \frac{k_{\mathrm{i}}}{k_{\mathrm{f}}|f(Q)|^{2}} I(\mathbf{Q}, E)$,

where $k_{\mathrm{i}}$ and $k_{\mathrm{f}}$ are the incident and final neutron wave vectors, and $f(Q)$ is the magnetic form factor of $\mathrm{Pr}^{3+}$ in the dipole approximation. For the sake of clarity, the raw data (Fig. 3a, b, e) have been symmetrized using the space group symmetry of $\mathrm{PrTiNbO}_{6}$ in the reciprocal lattice space (see Fig. 3c, d).

\section{Quantum many-body calculations}

We employ TRG approach for the accurate computation of equilibrium thermodynamics and spectral function of the RTIC model. Thermal TRG approach $^{34,51,52}$ is exploited to obtain the matrix product operator (MPO) representation of the thermal density matrix $\rho(\beta)=e^{-\beta \mathcal{H}}$, from which the thermodynamic properties, e.g., $C_{m}$ and $X$, can be evaluated. We introduce the distribution of couplings and fields along the chain (with length up to $I=1000$ ), and the observables are evaluated over 20 independent chain samples that have led to fully converged results of RTIC $\left(X_{j}^{\text {cal }}\right)$. In both thermodynamic and dynamic calculations, up to $D=200$ bond states are kept in MPO, which corresponds to negligible (machine-precision) truncation errors throughout and thus assuring a very high accuracy. We fit the reported thermodynamic data ${ }^{31}$ by minimizing the following loss function,

$R_{p}=\sqrt{\frac{1}{N_{0}} \sum_{j=1}^{N_{0}}\left(\frac{X_{j}^{\text {obs }}-X_{j}^{\text {cal }}}{\sigma_{j}^{\text {obs }}}\right)^{2}}$,

where $N_{0}, X_{j}^{\text {obs }}$, and $\sigma_{j}^{\text {obs }}$ are the sample number, measured value, and standard deviation of the experimental data, respectively.

Given the thermal density matrix $\rho(\beta)$, we perform real-time evolution and compute the dynamical spin structure factor ${ }^{53}$. We employ timedependent algorithm at finite temperature to calculate the timedependent correlation

$$
\left\langle S_{j}^{z}(t) S_{0}^{z}\right\rangle_{\beta}=\operatorname{Tr}\left[g_{j} S_{j}^{z} \cdot\left(e^{-i t \mathcal{H}} g_{0} S_{0}^{z} e^{-\beta \mathcal{H}} e^{i t \mathcal{H}}\right)\right],
$$

between $S_{j}^{z}$ (at time $t$ ) and $S_{0}{ }^{z}$ at time 0 and center of the chain. The last four terms are bracketed (computed first), so as to eliminate unnecessary entanglement accumulation in the course of time evolution. Given that, the dynamical spin structure factor can be computed via the Fourier transformation

$S(K, \omega)=\sum_{j=1}^{l} e^{-i \pi K j} \int_{-\infty}^{\infty}\left\langle S_{j}^{z}(t) S_{0}^{z}\right\rangle_{\beta} e^{i \omega t} d t$.

In practice, we perform dynamical computations on spin chains with length $=48$, over 40 samples. The real-time evolution is up to time $t / J=$ 10, guaranteeing a sufficient energy resolution below the instrumental resolution. The simulations are performed on spin chains with open boundary conditions, which, rendering no essential finite-size/boundary effects in the calculated spectral data of RTIC, as a short correlation length $\xi_{b}<<l b / 2$ is observed up to $t / J \leq 10$.

Besides, we have also carried out the ED calculations of 12 sites under periodic boundary conditions (see model no.3), which also shows good consistency with large-scale many-body calculations. The international system of units is used throughout this paper, and $\diamond$ represents thermal and/or sample average.

\section{DATA AVAILABILITY}

The datasets generated during and/or analyzed during the current study are available from the corresponding authors on reasonable request.

Received: 21 October 2020; Accepted: 28 January 2021; Published online: 31 March 2021

\section{REFERENCES}

1. Imry, Y. \& Ma, S.-k Random-field instability of the ordered state of continuous symmetry. Phys. Rev. Lett. 35, 1399-1401 (1975).

2. Harris, A. B. Effect of random defects on the critical behavior of Ising models. J. Phys. C: Solid State Phys. 7, 1671-1692 (1974).

3. Anderson, P. W. Absence of diffusion in certain random lattices. Phys. Rev. 109, 1492-1505 (1958).

4. Evers, F. \& Mirlin, A. D. Anderson transitions. Rev. Mod. Phys. 80, 1355-1417 (2008).

5. Vojta, T. Rare region effects at classical, quantum and nonequilibrium phase transitions. J. Phys. A: Math. Gen. 39, R143-R205 (2006). 
6. Vojta, T. Quantum Griffiths effects and smeared phase transitions in metals: Theory and experiment. J. Low. Temp. Phys. 161, 299-323 (2010).

7. Vojta, T. Disorder in quantum many-body systems. Annu. Rev. Condens. Matter Phys. 10, 233-252 (2019).

8. Fisher, D. S. Random transverse field Ising spin chains. Phys. Rev. Lett. 69, 534-537 (1992).

9. Fisher, D. S. Critical behavior of random transverse-field Ising spin chains. Phys. Rev. B 51, 6411-6461 (1995).

10. McCoy, B. M. \& Wu, T. T. Random impurities as the cause of smooth specific heats near the critical temperature. Phys. Rev. Lett. 21, 549-551 (1968).

11. Griffiths, R. B. Nonanalytic behavior above the critical point in a random Ising ferromagnet. Phys. Rev. Lett. 23, 17-19 (1969).

12. Young, A. P. \& Rieger, H. Numerical study of the random transverse-field Ising spin chain. Phys. Rev. B 53, 8486-8498 (1996).

13. Young, A. P. Finite-temperature and dynamical properties of the random transverse-field Ising spin chain. Phys. Rev. B 56, 11691-11700 (1997).

14. Iglói, F. \& Rieger, H. Random transverse Ising spin chain and random walks. Phys. Rev. B 57, 11404-11420 (1998).

15. Iglói, F. Exact renormalization of the random transverse-field Ising spin chain in the strongly ordered and strongly disordered Griffiths phases. Phys. Rev. B 65, 064416 (2002).

16. Ubaid-Kassis, S., Vojta, T. \& Schroeder, A. Quantum Griffiths phase in the weak itinerant ferromagnetic alloy $\mathrm{Ni}_{1-x} \mathrm{~V}_{x}$. Phys. Rev. Lett. 104, 066402 (2010).

17. Wang, R. et al. Quantum Griffiths phase inside the ferromagnetic phase of $\mathrm{Ni}_{1-x} \mathrm{~V}_{x}$ Phys. Rev. Lett. 118, 267202 (2017).

18. Xing, Y. et al. Quantum Griffiths singularity of superconductor-metal transition in Ga thin films. Science 350, 542-545 (2015).

19. Mohan, P. et al. Anomalously elastic intermediate phase in randomly layered superfluids, superconductors, and planar magnets. Phys. Rev. Lett. 105, 085301 (2010).

20. Pekker, D., Refael, G. \& Demler, E. Finding the elusive sliding phase in the superfluid-normal phase transition smeared by $c$-axis disorder. Phys. Rev. Lett. 105, 085302 (2010)

21. Coldea, R. et al. Quantum criticality in an Ising chain: experimental evidence for emergent $E_{8}$ symmetry. Science 327, 177-180 (2010).

22. Cui, Y. et al. Quantum criticality of the Ising-like screw chain antiferromagnet $\mathrm{SrCO}_{2} \mathrm{~V}_{2} \mathrm{O}_{8}$ in a transverse magnetic field. Phys. Rev. Lett. 123, 067203 (2019).

23. Wu, W., Bitko, D., Rosenbaum, T. F. \& Aeppli, G. Quenching of the nonlinear susceptibility at a $T=0$ spin glass transition. Phys. Rev. Lett. 71, 1919-1922 (1993)

24. Bitko, D., Rosenbaum, T. F. \& Aeppli, G. Quantum critical behavior for a model magnet. Phys. Rev. Lett. 77, 940-943 (1996).

25. Brooke, J., Bitko, D., Rosenbaum, T. F. \& Aeppli, G. Quantum annealing of a disordered magnet. Science 284, 779-781 (1999)

26. Tabei, S. M. A., Gingras, M. J. P., Kao, Y.-J., Stasiak, P. \& Fortin, J.-Y. Induced random fields in the $\mathrm{LiHo}_{x} \mathrm{Y}_{1-x} \mathrm{~F}_{4}$ quantum Ising magnet in a transverse magnetic field. Phys. Rev. Lett. 97, 237203 (2006).

27. $\mathrm{He}, \mathrm{Z}$. et al. Crystal growth and magnetic properties of $\mathrm{BaCO}_{2} \mathrm{~V}_{2} \mathrm{O}_{8}$. Chem. Mater. 17, 2924-2926 (2005)

28. Kimura, $\mathrm{S}$. et al. Collapse of magnetic order of the quasi one-dimensional Isinglike antiferromagnet $\mathrm{BaCO}_{2} \mathrm{~V}_{2} \mathrm{O}_{8}$ in transverse fields. J. Phys. Soc. Jpn. 82, 033706 (2013)

29. Faure, Q. et al. Topological quantum phase transition in the Ising-like antiferromagnetic spin chain $\mathrm{BaCO}_{2} \mathrm{~V}_{2} \mathrm{O}_{8}$. Nat. Phys. 14, 716-722 (2018).

30. Wang, Z. et al. From confined spinons to emergent fermions: observation of elementary magnetic excitations in a transverse-field Ising chain. Phys. Rev. B 94, 125130 (2016).

31. $\mathrm{Li}, \mathrm{Y}$. et al. Gapped ground state in the zigzag pseudospin-1/2 quantum antiferromagnetic chain compound $\mathrm{PrTiNbO}_{6}$. Phys. Rev. B 97, 184434 (2018).

32. Li, Y. et al. Partial up-up-down order with the continuously distributed order parameter in the triangular antiferromagnet $\mathrm{TmMgGaO}_{4}$. Phys. Rev. X 10, 011007 (2020).

33. Shen, Y. et al. Intertwined dipolar and multipolar order in the triangular-lattice magnet $\mathrm{TmMgGaO}_{4}$. Nat. Commun. 10, 4530 (2019).

34. $\mathrm{Li}, \mathrm{H}$. et al. Kosterlitz-thouless melting of magnetic order in the triangular quantum Ising material $\mathrm{TmMgGaO}_{4}$. Nat. Commun. 11, 1111 (2020)

35. Li, Y. et al. Crystalline electric-field randomness in the triangular lattice spin-liquid $\mathrm{YbMgGaO}_{4}$. Phys. Rev. Lett. 118, 107202 (2017)

36. Onoda, S. \& Tanaka, Y. Quantum melting of spin ice: Emergent cooperative quadrupole and chirality. Phys. Rev. Lett. 105, 047201 (2010).

37. Onoda, S. \& Tanaka, Y. Quantum fluctuations in the effective pseudospin-1/2 model for magnetic pyrochlore oxides. Phys. Rev. B 83, 094411 (2011).

38. Hayano, R. S. et al. Zero-and low-field spin relaxation studied by positive muons. Phys. Rev. B 20, 850-859 (1979).
39. Foronda, F. R. et al. Anisotropic local modification of crystal field levels in Prbased pyrochlores: a muon-induced effect modeled using density functional theory. Phys. Rev. Lett. 114, 017602 (2015).

40. Zorko, A. et al. Ground state of the easy-axis rare-earth kagome langasite $\mathrm{Pr}_{3} \mathrm{Ga}_{5} \mathrm{SiO}_{14}$. Phys. Rev. Lett. 104, 057202 (2010).

41. Li, Y. et al. Muon spin relaxation evidence for the $U(1)$ quantum spin-liquid ground State in the triangular antiferromagnet $\mathrm{YbMgGaO}_{4}$. Phys. Rev. Lett. 117, 097201 (2016).

42. $\mathrm{Li}, \mathrm{Y} . \mathrm{YbMgGaO}_{4}: \mathrm{A}$ triangular-lattice quantum spin liquid candidate. $A d v$. Quantum Technol. 2, 1900089 (2019).

43. Xu, G., Xu, Z. \& Tranquada, J. M. Absolute cross-section normalization of magnetic neutron scattering data. Rev. Sci. Instrum. 84, 083906 (2013).

44. Zaliznyak, I. A. \& Lee, S.-H. Magnetic Neutron Scattering, Tech. Rep. (Brookhaven National Laboratory, US, 2004).

45. $\mathrm{Li}, \mathrm{Y}$. et al. Nearest-neighbour resonating valence bonds in $\mathrm{YbMgGaO}_{4}$. Nat Commun. 8, 15814 (2017).

46. Li, Y. et al. Rearrangement of uncorrelated valence bonds evidenced by lowenergy spin excitations in $\mathrm{YbMgGaO}_{4}$. Phys. Rev. Lett. 122, 137201 (2019).

47. Cevallos, A. F., Stolze, K., Kong, T. \& Cava, R. J. Anisotropic magnetic properties of the triangular plane lattice material $\mathrm{TmMgGaO}_{4}$. Mater. Res. Bull. 105, 154-158 (2018).

48. Hillier, A. D., King, P. J. C., Cottrell, S. P. \& Lord, J. S. The MuSR User Guide. (ISIS Facility, STFC, Rutherford Appleton Laboratory, Oxford, England, 2005).

49. Bewley, R. I. et al. MERLIN, a new high count rate spectrometer at ISIS. Phys. $B$ (Amst.) 385, 1029-1031 (2006).

50. Bewley, R. I., Taylor, J. W. \& Bennington, S. M. LET, a cold neutron multi-disk chopper spectrometer at ISIS. Nucl. Instr. Meth. Phys. 637, 128-134 (2011).

51. Li, W. et al. Linearized tensor renormalization group algorithm for the calculation of thermodynamics of quantum lattice models. Phys. Rev. Lett. 106, 127202 (2011).

52. Chen, B.-B. et al. Exponential thermal tensor network approach for quantum lattice models. Phys. Rev. X 8, 031082 (2018).

53. Barthel, T., Schollwöck, U. \& White, S. R. Spectral functions in one-dimensional quantum systems at finite temperature using the density matrix renormalization group. Phys. Rev. B 79, 245101 (2009).

\section{ACKNOWLEDGEMENTS}

We thank P. Gegenwart, Jian Cui, and A.A. Tsirlin for helpful discussions. Experiments at the ISIS Pulsed Neutron and Muon Source were supported by a beamtime allocation from the Science and Technology Facilities Council. The work in Augsburg was supported by the German Science Foundation through TRR- 80 and the German Federal Ministry for Education and Research through the Sofja Kovalevskaya Award of the Alexander von Humboldt Foundation. Q.-Y.L., T.L., and W.L. acknowledge the NSFC through Grant Nos. 11974036, 11834014, 11704113. Y.L. was supported by the Fundamental Research Funds for the Central Universities, HUST: 2020kfyXJJS054.

\section{AUTHOR CONTRIBUTIONS}

Y.L. and D.A. planned the experiments. Y.L. synthesized and characterized the sample. Y.L., D.J.V., and D.A. collected the neutron scattering data. Y.L. and P.K.B. collected the muon spin relaxation data. Y.L. analyzed the experimental data and performed the exact diagonalization calculations. Q.Y.L. carried out the large-scale quantum manybody calculations, with the guidance from W.L. and T.L. Y.L. and W.L. wrote the manuscript with comments from all co-authors.

\section{COMPETING INTERESTS}

The authors declare no competing interests.

\section{ADDITIONAL INFORMATION}

Supplementary information The online version contains supplementary material available at https://doi.org/10.1038/s41535-021-00333-6.

Correspondence and requests for materials should be addressed to Y.L. or W.L.

Reprints and permission information is available at http://www.nature.com/ reprints

Publisher's note Springer Nature remains neutral with regard to jurisdictional claims in published maps and institutional affiliations. 
Attribution 4.0 International License, which permits use, sharing, adaptation, distribution and reproduction in any medium or format, as long as you give appropriate credit to the original author(s) and the source, provide a link to the Creative Commons license, and indicate if changes were made. The images or other third party material in this article are included in the article's Creative Commons license, unless indicated otherwise in a credit line to the material. If material is not included in the article's Creative Commons license and your intended use is not permitted by statutory regulation or exceeds the permitted use, you will need to obtain permission directly from the copyright holder. To view a copy of this license, visit http://creativecommons. org/licenses/by/4.0/.

(c) The Author(s) 2021 